\title{
Severe prolonged neutropenia following administration of tocilizumab in a patient affected by COVID-19: a case report and brief review of the literature
}

\author{
Luca Bernardo $^{1}$ - Stefania Del Sesto ${ }^{2,3}$. Laura Giordano ${ }^{1}$. Anna Rita Benincaso ${ }^{1}$ - Piero Biondi ${ }^{1}$ Vinicio Goj ${ }^{1}$. \\ Francesca Ferrara ${ }^{1} \cdot$ Antonella Talenti $^{1} \cdot$ Antonella Grisolia $^{4} \cdot$ Paolo Antonio Ascierto ${ }^{5}$
}

Published online: 14 September 2020

(c) The Author(s) 2020

\begin{abstract}
Tocilizumab is one of the newest therapeutic options for the acute respiratory distress syndrome (ARDS) caused by the recently discovered severe acute respiratory syndrome coronavirus 2 (SARS-CoV-2) $\beta$-coronavirus. Several trials are currently ongoing to assess the efficacy and safety profile of tocilizumab in treating ARDS. In this article, we present the case of a Black patient with acute pneumonia who benefited greatly from tocilizumab, but developed severe prolonged neutropenia. Considering the increasing use of tocilizumab among patients with coronavirus disease 2019 (COVID-19), this case warrants further research regarding the possible adverse hematological effects that need to be monitored in order to prevent secondary infections.
\end{abstract}

\section{Introduction}

Coronavirus disease 2019 (COVID-19), an emergent pandemic infection caused by the novel $\beta$-coronavirus severe acute respiratory syndrome coronavirus 2 (SARS-CoV-2), primarily targets the lungs but also affects the heart and kidneys [1]. In addition to mild respiratory flu-like symptoms, SARS-CoV-2 may cause severe acute respiratory distress syndrome (ARDS), sepsis, or multiorgan dysfunction [1, 2], as seen in the other two previous $\beta$-coronavirus outbreaks [severe acute respiratory syndrome (SARS) and Middle East respiratory syndrome (MERS)] [3, 4].

The exact pathogenesis of such life-threatening manifestations of $\beta$-coronavirus infections is currently unknown but

Stefania Del Sesto

stefania.delsesto@unimi.it

1 Department of Pediatrics, Fatebenefratelli e Oftalmico Hospital, Milan, Italy

2 Department of Pediatrics, Vittore Buzzi Children's Hospital, Milan, Italy

3 Università Degli Studi Di Milano, Milan, Italy

4 Department of Infectious Diseases, Luigi Sacco Hospital, Milan, Italy

5 Istituto Nazionale Tumori IRCCS Fondazione "G. Pascale", Naples, Italy a massive immune host response - the so-called "cytokine storm'-is considered the most likely cause, as described in cases of ARDS in patients with SARS or MERS [5-7]. A dysregulation of the immune system [i.e. cytokine release syndrome (CRS)], by means of an uncontrollable release of proinflammatory cytokines, is also described in the current pandemic, especially in severely ill patients $[1,8]$. Among these cytokines, interleukin (IL)-6 has a key role in the proinflammatory cascade. It is mainly produced in response to inflammatory tissue damage $[9,10]$.

Excessive IL-6 levels lead to a multitude of functional and anatomical changes, finally inducing matrix deposition in targeted tissues, which may result in alveolar-capillary membrane dysfunction and increased vascular permeability [11].

In patients with multiorgan dysfunction affected by ARDS, IL-6 levels are known to be related to a worse prognosis [12]. Even in the context of COVID-19-related ARDS, high levels of ferritin and IL-6 have been reported as significant predictors of mortality [13]. Thus, immunosuppression could be a valid therapeutic option for the hyperinflammatory status described in COVID-19, particularly by blocking the IL-6 pathway $[14,15]$.

Tocilizumab, one of the most commonly used IL-6 receptor blockers, is primarily used to treat rheumatic diseases and post-immunotherapy CRS $[16,17]$. In these situations, tocilizumab is generally well tolerated. In keeping with previous 
research, a recent double-blind, randomized controlled trial in patients with rheumatoid arthritis (RA) reported the main adverse events associated with tocilizumab were a transient decrease in leukocytes, increase in liver enzymes, dyslipidemia, and infection-related adverse events [18].

In this article, we report the case of a patient with respiratory failure related to COVID-19 who had a rapid favorable outcome after two doses of tocilizumab, but developed severe prolonged neutropenia. Written informed consent was obtained from the patient prior to the publication of this case report.

\section{Description of the case}

A Black 65-year-old man presented to our emergency department with a few days' history of fever, complaining of a presyncopal episode. Although past medical history revealed the presence of hypertensive cardiomyopathy, paroxystic atrial fibrillation, and chronic stage II renal insufficiency, he was not taking any medications due to poor compliance.

Upon presentation, his physical examination was unremarkable. Vital signs showed oxygen saturation of $99 \%$ on room air, heart rate of 83 beats/min, blood pressure of $160 / 80 \mathrm{mmHg}$, and a body temperature of $38.1{ }^{\circ} \mathrm{C}$.

Blood tests showed mild leukocytosis $\left(10.9 \times 10^{9} / \mathrm{L}\right)$ with left shift (neutrophils $9 \times 10^{9} / \mathrm{L}$ ), increased C-reactive protein [CRP; $89.8 \mathrm{mg} / \mathrm{L}, 9 \times$ the upper limit of normal (ULN)], slightly elevated creatinine $(1.53 \mathrm{mg} / \mathrm{dL})$, and normal urea and liver function tests. The chest X-ray was compatible with mild bilateral interstitial pneumonia (Fig. 1). Real-time reverse transcription polymerase chain reaction (RT-PCR) on a nasopharyngeal swab was positive for SARS-CoV-2.

Treatment with ceftriaxone and clarithromycin was initiated due to concerns that the patient had a superimposed bacterial lung infection.

Blood investigations on day 3 revealed persistently increased CRP levels $(91 \mathrm{mg} / \mathrm{L})$; therefore, antibiotic treatment was switched from ceftriaxone to piperacillin-tazobactam, and combination therapy with hydroxychloroquine and lopinavir-ritonavir was started and continued for 5 days.

During hospitalization, the patient's clinical conditions worsened, with the appearance of type 1 (hypoxemic) respiratory failure. Blood gas analysis on room air reported mild respiratory alkalosis with hypoxemia $(\mathrm{pH} 7.44$, partial pressure of oxygen $60 \mathrm{mmHg}$, partial pressure of carbon dioxide $34 \mathrm{mmHg}$, ratio of arterial oxygen partial pressure to fractional inspired oxygen 295, alveolar-arterial oxygen gradient $45 \mathrm{mmHg}$, and normal lactate and bicarbonate). Upon physical examination, the patient was tachypneic and still febrile; oxygen saturation dropped at $92 \%$ on room air and fine diffuse crackles could be heard on lung auscultation.

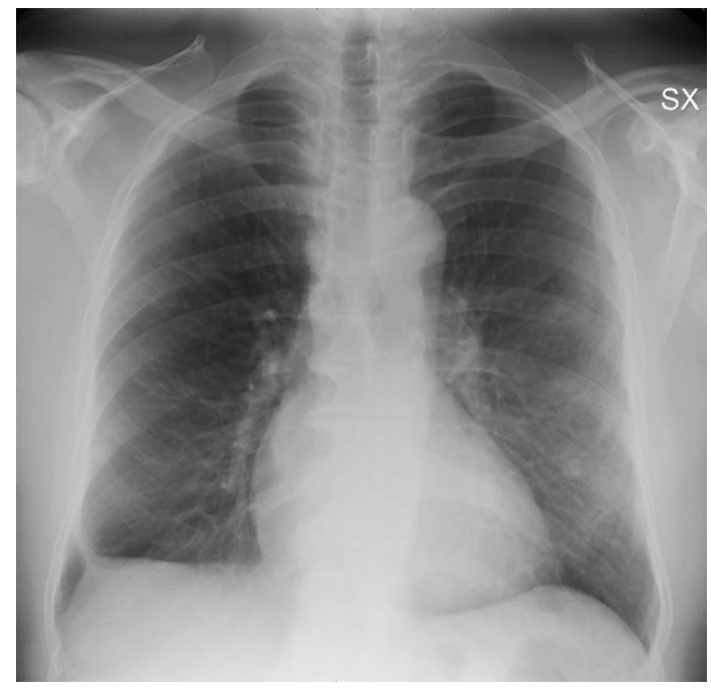

Fig. 1 Chest radiograph showing mild opacities in the perihilar lung fields bilaterally and linear opacity in the right inferior lobe

Laboratory tests showed leukocytosis $\left(10.99 \times 10^{9} / \mathrm{L}\right)$ with neutrophilia $\left(9.8 \times 10^{9} / \mathrm{L}\right)$ and subsequent lymphopenia $\left(1.19 \times 10^{9} / \mathrm{L}\right)$, a much increased CRP level $(337 \mathrm{mg} / \mathrm{L})$, microcytic anemia (hemoglobin $10 \mathrm{mg} / \mathrm{dL}$, mean corpuscular volume 72.7 femtoliters), prolonged prothrombin time (international normalized ratio 1.57), and a stable creatinine level.

A chest computed tomography (CT) scan was performed, showing bilateral diffuse lung consolidations and groundglass opacities, mainly in posterior locations. Mild pleural effusion and mediastinal lymphadenopathy were also present (Fig. 2a).

Because of a lack of response to previous pharmacological therapy, after infectious disease screening tests and evaluation of IL-6 levels (which resulted in $>10 \times \mathrm{ULN}$ ), the patient was administered two intravenous doses of tocilizumab $8 \mathrm{mg} / \mathrm{kg}$ administered $12 \mathrm{~h}$ apart (day 8 after admission).

Soon after, the patient's clinical condition began to improve with immediate defervescence. After $48 \mathrm{~h}$, he no longer required oxygen supplementation. Blood investigations showed a rapid decrease in his CRP level $(96.40 \mathrm{mg} / \mathrm{L})$ and white blood cell count $\left(2.36 \times 10^{9} / \mathrm{L}\right)$, particularly his absolute neutrophil count (ANC, $0.89 \times 10^{9} / \mathrm{L}$ ), and improvement in his hemoglobin and creatinine levels.

A second chest CT scan was performed a week later, documenting marked improvement of lung transparency, with important reduction of ground-glass opacities and consolidations. Some ground-glass opacities were still present, distributed mainly in a posterior subpleural location (Fig. 2b).

Two days after administration of tocilizumab, the patient's blood work showed persistence of leukopenia $\left(2.32 \times 10^{9} / \mathrm{L}\right)$, with mild lymphopenia $\left(1.06 \times 10^{9} / \mathrm{L}\right)$ and progression of 


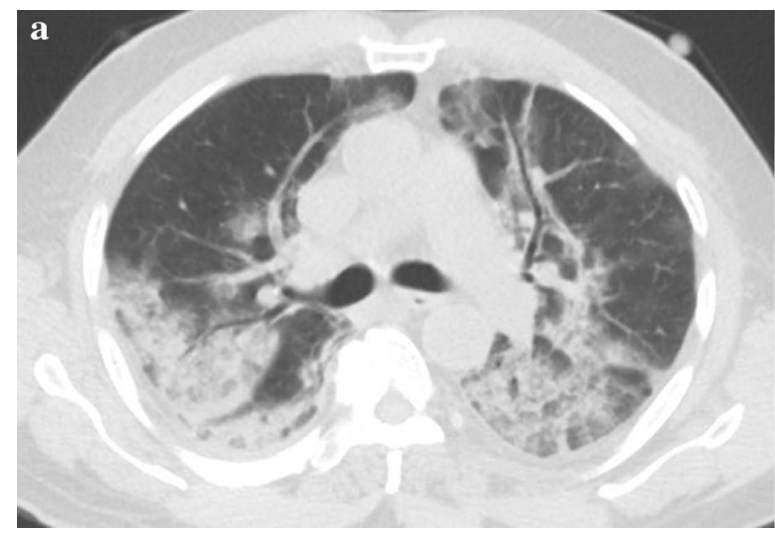

Fig. 2 a (left) Unenhanced chest CT scan in a transverse section showing the presence of diffuse ground-glass opacities and consolidations, with prevalent posterior distribution; some linear, mainly peripheral, opacities; mild pleural effusion. b (right) Unenhanced

neutropenia $\left(0.52 \times 10^{9 /} \mathrm{L}\right)$. The hematologic consultant advised that no hematologic treatment was required unless a superimposed infection was suspected.

Serial blood examinations showed worsening of neutropenia; nadir $\left(0.25 \times 10^{9} / \mathrm{L}\right)$ was reached 12 days after the tocilizumab injections. Severe neutropenia lasted for at least 4 weeks; on day 30 after the tocilizumab injections, the patient's ANC began to improve $\left(0.62 \times 10^{9} / \mathrm{L}\right)$, although considerably far from normal values $\left(\geq 1.5 \times 10^{9} / \mathrm{L}\right)$.

\section{Discussion}

Tocilizumab is a recombinant humanized monoclonal antibody, directed against the IL-6 receptor. In the USA and EU, tocilizumab is approved for the treatment of RA, juvenile idiopathic arthritis, giant cell arteritis, and chimeric antigen receptor T-cell therapy-induced CRS. Phase II and III studies are ongoing in order to extend the indications to other chronic inflammatory diseases $[16,17]$.

Tolerance of tocilizumab has mainly been investigated regarding its use in rheumatologic diseases. According to a systematic review of biologic agents in any disease (except HIV/AIDS) that included eight randomized placebo-controlled trials of tocilizumab, there were no significant differences between tocilizumab and placebo in terms of incidence of serious adverse events, upper respiratory infections, or withdrawals due to adverse events [19]. However, patients treated with tocilizumab were more likely to develop dyslipidemia, gastrointestinal disorders, rashes, or decreased neutrophil counts [19].

In a cumulative analysis of almost 5 years of tocilizumab exposure in five clinical trials that involved patients with moderate to severe RA, infections were the most common adverse event. The rate of serious infections was

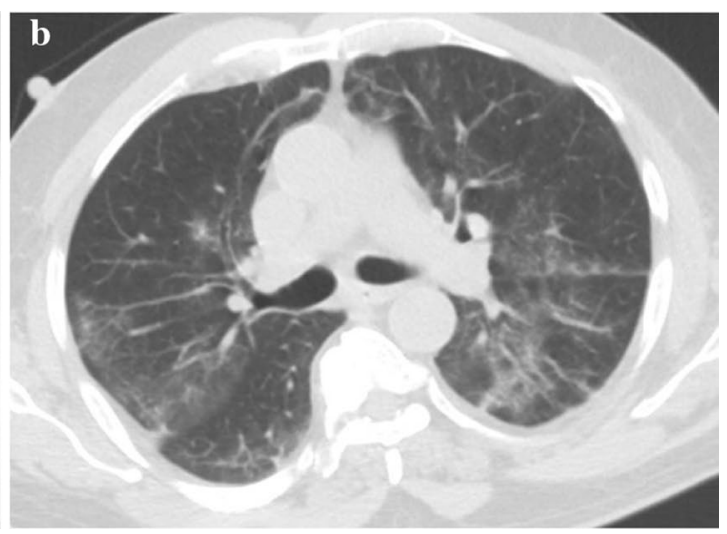

chest CT scan in a transverse section showing marked improvement of lung consolidations; some peripheral and posterior linear and ground-glass opacities are still visible. $C T$ computed tomography

4.5/100 patient-years [20]. A meta-analysis of six randomized clinical trials found a higher risk of infections within the group assigned to $8 \mathrm{mg} / \mathrm{kg}$ tocilizumab plus methotrexate rather than the group assigned to methotrexate alone [21].

Among laboratory changes, neutropenia has been reported as one of the most frequent adverse events following tocilizumab injection [22]. Neutropenia is defined as an $\mathrm{ANC}<1.5 \times 10^{9} / \mathrm{L}$ in adults. It is classified as mild [grade 2 using Common Terminology Criteria for Adverse Events (CTCAE)] if the ANC is between 1.0 and $1.5 \times 10^{9} / \mathrm{L}$, moderate (CTCAE grade 3 ) between 0.5 and $1.0 \times 10^{9} / \mathrm{L}$, and severe (CTCAE grade 4 ) if $<0.5 \times 10^{9} / \mathrm{L}$ [23]. Severe neutropenia is among the few indications to cease treatment with tocilizumab in order not to further increase the risk of infections. CTCAE grade 3 neutropenia is an indication to temporarily suspend tocilizumab, which can be resumed after 4 weeks of interruption in case of remission of neutropenia [17].

A Japanese analysis of patients with RA receiving monotherapy with tocilizumab reported CTCAE grade 2 and grade 3 neutropenia in $15 \%$ and $6 \%$ of patients, respectively [24]. In the pooled analysis of five long-term clinical trials of tocilizumab in patients with RA receiving tocilizumab alone or in combination with methotrexate, $4.8 \%$ of patients developed grade 3 neutropenia, and $0.7 \%$ developed grade 4 neutropenia, which resolved spontaneously or after withdrawal of the drug. None of the patients with grade 4 neutropenia experienced serious infections within 30 days of observation [20].

Another analysis of pooled data from placebo-controlled trials of tocilizumab in patients with RA showed that the incidence of serious infections in patients treated with tocilizumab did not correlate with the ANCs. Moreover, no serious infections occurred in patients with grade 3 or 4 
ANCs, which were indications to interrupt or discontinue tocilizumab [25].

Different pathogenetic mechanisms of tocilizumabinduced neutropenia have been proposed, from myelosuppression to proapoptotic effect to extravascular margination of circulating neutrophils [25].

In a recent pharmacodynamic study, 18 healthy individuals were randomized to receive tocilizumab $8 \mathrm{mg} / \mathrm{kg}$ or placebo [26]. After tocilizumab administration, the kinetic profile of neutrophils was consistent with their major retention in the spleen and liver, although the functional profile of neutrophils showed no differences in surface activation markers or apoptosis rates between tocilizumab and placebo; however, none of the participants experienced grade 3 or 4 neutropenia [26].

In the context of the ongoing pandemic, Xu et al. [15] were among the first who thought that tocilizumab could have a possible beneficial effect on CRS provoked by SARSCoV-2. In February 2020, they treated 21 patients affected by severe or critical COVID-19 with tocilizumab. Oxygen supplementation was reduced in $75 \%$ of patients and CT images improved in $90.5 \%$ [15].

Published data from China regarding the safety profile of tocilizumab in COVID-19 did not report any serious adverse events, in particular neither infections nor neutropenia [15], whereas Italian publications have reported a possible increased risk of superimposed infections and candidemia $[27,28]$.

Several clinical trials are currently evaluating the efficacy and safety of tocilizumab in SARS-CoV-2 pneumonia [29-33]. Preliminary results from an Italian study [33] are encouraging, but not definitive, in terms of reductions in mortality. No new adverse events were reported [33].

Black people are known to have lower ANCs and a higher prevalence of neutropenia than other ethnicities [34]. Data from $>25,000$ people participating in a US National Health and Nutritional Examination Survey from 1999 to 2004 showed that $4.50 \%$ of Black participants had neutropenia, whereas this was seen in only $0.79 \%$ of White participants, and $0.38 \%$ of Mexican American participants [34]. Etiology of neutropenia in Black people has in part been discovered, as genetic polymorphisms have been found in association to lower neutrophil counts in the so-called benign ethnic neutropenia (BEN). Reports about the natural history of BEN in adults are actually lacking. Only one recent prospective analysis of 46 participants of African ancestry having different grades of BEN, showed normal rates of common infections; however, grade 4 neutropenia was rarely represented [35].

In our experience of the use of tocilizumab in five patients with COVID-19, two patients, both Black Africans, developed neutropenia. We have presented the case of one of these patients, whose neutropenia was severe and prolonged. Although a causal relation cannot be demonstrated from the observation of a single case, we believe that a concomitant genetic factor (as stated for BEN) could have enhanced the neutropenic effect of tocilizumab in this patient.

\section{Take home messages}

- Neutropenia is one of the most frequent laboratory changes following administration of tocilizumab.

- Patients receiving tocilizumab who develop neutropenia need to be followed-up and screened for secondary infections.

- Black patients are more likely to have lower neutrophil counts than patients of other ethnicities due to a genetic predisposition.

- A concomitant genetic factor could enhance the neutropenic effect of tocilizumab.

\section{Declarations}

Funding Open access funding provided by Università degli Studi di Milano within the CRUI-CARE Agreement.

Conflict of interest Luca Bernardo, Stefania Del Sesto, Laura Giordano, Anna Rita Benincaso, Piero Biondi, Vinicio Goj, Francesca Ferrara, Antonella Talenti, Antonella Grisolia, and Paolo Antonio Ascierto declare no conflicts of interest.

Ethics approval Not applicable.

Consent to participate Not applicable.

Consent for publication Written consent was obtained from the patient prior to publication of this article.

Data availability Not applicable.

Code availability Not applicable.

Author contributions LB conceptualised the case report and collected the data. SDS reviewed the literature and drafted the manuscript. ARB and LG contributed to the literature review and drafting of the manuscript. AG helped with data collection and revising the manuscript. PB, $\mathrm{VG}, \mathrm{FF}$ and AT analyzed the data and revised the manuscript. PAA critically reviewed and corrected the manuscript. All authors read and approved the submitted version of the article.

Open Access This article is licensed under a Creative Commons Attribution-NonCommercial 4.0 International License, which permits any non-commercial use, sharing, adaptation, distribution and reproduction in any medium or format, as long as you give appropriate credit to the original author(s) and the source, provide a link to the Creative Commons licence, and indicate if changes were made. The images or other third party material in this article are included in the article's Creative Commons licence, unless indicated otherwise in a credit line to the material. If material is not included in the article's Creative Commons licence and your intended use is not permitted by statutory regulation or exceeds the permitted use, you will need to obtain permission 
directly from the copyright holder. To view a copy of this licence, visit http://creativecommons.org/licenses/by-nc/4.0/.

\section{References}

1. Huang C, Wang Y, Li X, et al. Clinical features of patients infected with 2019 novel coronavirus in Wuhan, China. Lancet. 2020;395(10223):497-506.

2. Yang X, Yu Y, Xu J, et al. Clinical course and outcomes of critically ill patients with SARS-CoV-2 pneumonia in Wuhan, China: a single-centered, retrospective, observational study. Lancet Respir Med. 2020;8(5):475-81 (published erratum appears in Lancet Respir Med. 2020;8(4):e26).

3. Lee $\mathrm{N}$, Hui $\mathrm{D}, \mathrm{Wu} \mathrm{A}$, et al. A major outbreak of severe acute respiratory syndrome in Hong Kong. N Engl J Med. 2003;348(20):1986-94.

4. Assiri A, Al-Tawfiq JA, Al-Rabeeah AA, et al. Epidemiological, demographic, and clinical characteristics of 47 cases of Middle East respiratory syndrome coronavirus disease from Saudi Arabia: a descriptive study. Lancet Infect Dis. 2013;13(9):752-61.

5. Wong CK, Lam CW, Wu AK, et al. Plasma inflammatory cytokines and chemokines in severe acute respiratory syndrome. Clin Exp Immunol. 2004;136(1):95-103.

6. Channappanavar R, Perlman S. Pathogenic human coronavirus infections: causes and consequences of cytokine storm and immunopathology. Semin Immunopathol. 2017;39(5):529-39.

7. Mahallawi WH, Khabour OF, Zhang Q, et al. MERS-CoV infection in humans is associated with a pro-inflammatory Th1 and Th17 cytokine profile. Cytokine. 2018;104:8-13.

8. Zhang S, Li L, Shen A, et al. Rational use of tocilizumab in the treatment of novel coronavirus pneumonia. Clin Drug Investig. 2020;40(6):511-8.

9. Rincon M. Interleukin-6: from an inflammatory marker to a target for inflammatory diseases. Trends Immunol. 2012;33(11):571-7.

10. Qin C, Zhou L, Hu Z, et al. Dysregulation of immune response in patients with Coronavirus 2019 (COVID-19) in Wuhan, China. Clin Infect Dis. 2020;71(15):762-8.

11. Liu B, Li M, Zhou Z, et al. Can we use interleukin-6 (IL-6) blockade for coronavirus disease 2019 (COVID-19)-induced cytokine release syndrome (CRS)? J Autoimmun. 2020;111:102452.

12. Hui L, Zhang $X$, An $X$, et al. Higher serum procalcitonin and IL-6 levels predict worse diagnosis for acute respiratory distress syndrome patients with multiple organ dysfunction. Int J Clin Exp Pathol. 2017;10(7):7401-7.

13. Ruan Q, Yang K, Wang W, et al. Clinical predictors of mortality due to COVID-19 based on an analysis of data of 150 patients from Wuhan, China. Intensive Care Med. 2020;46(5):846-8 (published erratum appears in Intensive Care Med. 2020;46(6):1294-7).

14. Mehta P, McAuley DF, Brown M, et al. COVID-19: consider cytokine storm syndromes and immunosuppression. Lancet. 2020;395(10229):1033-4.

15. Xu X, Han M, Li T, et al. Effective treatment of severe COVID19 patients with tocilizumab. Proc Natl Acad Sci USA. 2020;117(20):10970-5.

16. Rubbert-Roth A, Furst DE, Nebesky JM, et al. A review of recent advances using tocilizumab in the treatment of rheumatic diseases. Rheumatol Ther. 2018;5(1):21-42.

17. Sheppard M, Laskou F, Stapleton PP, et al. Tocilizumab (Actemra). Hum Vaccin Immunother. 2017;13(9):1972-88.

18. Baek HJ, Lim MJ, Park W, et al. Efficacy and safety of tocilizumab in Korean patients with active rheumatoid arthritis. Korean J Intern Med. 2019;34(4):917-31.
19. Singh JA, Wells GA, Christensen R, et al. Adverse effects of biologics: a network meta-analysis and Cochrane overview. Cochrane Database Syst Rev. 2011;2011(2):CD008794.

20. Genovese MC, Rubbert-Roth A, Smolen JS, et al. Longterm safety and efficacy of tocilizumab in patients with rheumatoid arthritis: a cumulative analysis of up to 4.6 years of exposure. J Rheumatol. 2013;40(6):768-80.

21. Campbell L, Chen C, Bhagat SS, et al. Risk of adverse events including serious infections in rheumatoid arthritis patients treated with tocilizumab: a systematic literature review and meta-analysis of randomized controlled trials. Rheumatology (Oxford). 2011;50(3):552-62.

22. Shovman O, Shoenfeld Y, Langevitz P. Tocilizumab-induced neutropenia in rheumatoid arthritis patients with previous history of neutropenia: case series and review of literature. Immunol Res. 2015;61(1-2):164-8

23. Boxer LA. How to approach neutropenia. Hematology Am Soc Hematol Educ Program. 2012;2012:174-82.

24. Nishimoto N, Ito K, Takagi N. Safety and efficacy profiles of tocilizumab monotherapy in Japanese patients with rheumatoid arthritis: meta-analysis of six initial trials and five long-term extensions. Mod Rheumatol. 2010;20(3):222-32.

25. Moots RJ, Sebba A, Rigby W, et al. Effect of tocilizumab on neutrophils in adult patients with rheumatoid arthritis: pooled analysis of data from phase 3 and 4 clinical trials. Rheumatology (Oxford). 2017;56(4):541-9.

26. Lok LSC, Farahi N, Juss JK, et al. Effects of tocilizumab on neutrophil function and kinetics. Eur J Clin Invest. 2017;47(10):736-45.

27. Quartuccio L, Sonaglia A, McGonagle D, et al. Profiling COVID19 pneumonia progressing into the cytokine storm syndrome: results from a single Italian Centre study on tocilizumab versus standard of care. J Clin Virol. 2020;129:104444.

28. Antinori S, Bonazzetti C, Gubertini G, et al. Tocilizumab for cytokine storm syndrome in COVID-19 pneumonia: an increased risk for candidemia? Autoimmun Rev. 2020;19(7):102564.

29. Chinese Clinical Trial Registry. A multicenter, randomized controlled trial for the efficacy and safety of tocilizumab in the treatment of new coronavirus pneumonia (COVID-19). 2020. https ://www.chictr.org.cn/showprojen. aspx? proj=49409. Accessed 20 May 2020.

30. Tocilizumab in COVID-19 pneumonia (TOCIVID-19) US National Library of Medicine. ClinicalTrials.gov. 2020. https ://clinicaltrials.gov/ct2/show/NCT04317092. Accessed 20 May 2020.

31. ClinicalTrials.gov. Tocilizumab for SARS-CoV2 (COVID-19) severe pneumonitis [NCT04315480]. 2020. https://clinicaltrials. gov/ct2/show/NCT04315480. Accessed 20 May 2020.

32. ClinicalTrials.gov. A study to evaluate the safety and efficacy of tocilizumab in patients with severe COVID-19 pneumonia (COVACTA) [NCT04320615]. 2020. https://clinicaltrials.gov/ct2/show/ NCT04320615. Accessed 20 May 2020.

33. Agenzia Italiana del Farmaco. Tocivid-19 study: encouraging but not definitive results: (communication no. 598) [in Italian]. Rome: Agenzia Italiana del Farmaco; 5 May 2020.

34. Hsieh MM, Everhart JE, Byrd-Holt DD, et al. Prevalence of neutropenia in the U.S. population: age, sex, smoking status, and ethnic differences. Ann Intern Med. 2007;146(7):486-92.

35. Lakhotia R, Aggarwal A, Link ME, et al. Natural history of benign ethnic neutropenia in individuals of African ancestry. Blood Cells Mol Dis. 2019;77:12-6. 